\title{
Closed-Form Exact Solutions for the Unforced Quintic Nonlinear Oscillator
}

\author{
Augusto Beléndez, ${ }^{1,2}$ Enrique Arribas, ${ }^{3}$ Tarsicio Beléndez, ${ }^{1,2}$ Carolina Pascual, ${ }^{1,2}$ \\ Encarnación Gimeno, ${ }^{1,2}$ and Mariela L. Álvarez ${ }^{1,2}$ \\ ${ }^{1}$ Instituto Universitario de Física Aplicada a las Ciencias y las Tecnologías, Universidad de Alicante, Apartado 99, \\ 03080 Alicante, Spain \\ ${ }^{2}$ Departamento de Física, Ingeniería de Sistemas y Teoría de la Señal, Universidad de Alicante, Apartado 99, 03080 Alicante, Spain \\ ${ }^{3}$ Departamento de Física Aplicada, Universidad de Castilla-La Mancha, Avda. España s/n, 02071 Albacete, Spain
}

Correspondence should be addressed to Augusto Beléndez; a.belendez@ua.es

Received 6 November 2016; Accepted 5 January 2017; Published 2 February 2017

Academic Editor: Zhi-Yuan Sun

Copyright (C) 2017 Augusto Beléndez et al. This is an open access article distributed under the Creative Commons Attribution License, which permits unrestricted use, distribution, and reproduction in any medium, provided the original work is properly cited.

\begin{abstract}
Closed-form exact solutions for the periodic motion of the one-dimensional, undamped, quintic oscillator are derived from the first integral of the nonlinear differential equation which governs the behaviour of this oscillator. Two parameters characterize this oscillator: one is the coefficient of the linear term and the other is the coefficient of the quintic term. Not only the common case in which both coefficients are positive but also all possible combinations of positive and negative values of these coefficients which provide periodic motions are considered. The set of possible combinations of signs of these coefficients provides four different cases but only three different pairs of period-solution. The periods are given in terms of the complete elliptic integral of the first kind and the solutions involve Jacobi elliptic function. Some particular cases obtained varying the parameters that characterize this oscillator are presented and discussed. The behaviour of the periods as a function of the initial amplitude is analysed and the exact solutions for several values of the parameters involved are plotted. An interesting feature is that oscillatory motions around the equilibrium point that is not at $x=0$ are also considered.
\end{abstract}

\section{Introduction}

Mathematical models based on nonlinear oscillators have been widely used in physics, engineering, applied mathematics, and related fields $[1,2]$. These nonlinear systems have been the focus of attention for many years and several methods have been used to find approximate solutions to them $[1,3]$. In conservative nonlinear oscillators the restoring force is not dependent on time, the total energy is constant [1], and any oscillation is stationary. The aim of this paper is to obtain closed-form exact periodic solutions to the quintic equation corresponding to a nonlinear oscillator described by a differential equation with fifthpower nonlinearity. Due to the presence of the fifth-power nonlinearity, this oscillator is difficult to handle and has not been studied as extensively as the Duffing oscillator with cubic nonlinearity. For this reason, several techniques have been used to obtain analytical approximate expressions for the period and the solution of the quintic oscillator. Lin [4] proposed a new parameter iteration technique to solve the Duffing equation with strong and higher-order nonlinearity. Ramos [5] approximately solved the quintic equation using some Lindstedt-Poincaré techniques. Pirbodaghi et al. [6] obtained an accurate analytical approximate solution to Duffing equations with cubic and quintic nonlinearities using the homotopy analysis method and homotopy Padé technique. Wu et al. [7] approximately solved this nonlinear oscillator using a method that incorporates salient features of both Newton's method and the harmonic balance method. Later, Lai et al. [8] used a Newton-harmonic balancing approach to obtain accurate approximate analytical higherorder solutions for strong nonlinear Duffing oscillators with cubic-quintic restoring force. They also discussed the effect of strong quantic nonlinearity on accuracy as compared to cubic 
nonlinearity. Beléndez et al. [9] approximately solved the quintic oscillator using a cubication method which allowed them to obtain approximate analytical expressions for the period and the solution in terms of elementary functions. Scarpello and Ritelli [10] exactly solved the quintic oscillator, but only when the coefficient for the linear term is equal to one and the coefficient for the nonlinear term is positive. Elías-Zúñiga [11] derived the exact solution of the cubicquintic Duffing equation based on the use of Jacobi elliptic functions by the same method that he used to obtain the exact solution of the mixed-parity Helmholtz-Duffing oscillator [12]. However, in both cases he did not solve the nonlinear differential equation but assumed that its exact solution is given by a rational equation which includes the $\mathrm{cn}$ Jacobian elliptic function and five unknown parameters that need to be determined. Based on his pervious results, Elías-Zúñiga obtained the analytical approximate solution of the damped cubic-quintic Duffing oscillator [13] and also developed a "quintication" method [14] to obtain approximate analytical solutions of conservative nonlinear oscillators. Recently, Beléndez et al. [15] have exactly solved the unforced cubicquintic Duffing oscillator, providing exact expressions for the period and the solution, but only for oscillations around $x=0$ and taken into account that the coefficients for the linear and the nonlinear terns are positive.

In this paper we obtain the closed-form exact expressions for the period and the solution of the quintic Duffing nonlinear oscillator modelled by the second-order differential equation $d^{2} x / d t^{2}+a_{1} x+a_{5} x^{5}=0$, where $a_{1}$ and $a_{5}$ are the coefficients of the linear and the nonlinear terms, respectively, considering all possible combinations of signs of $a_{1}$ and $a_{5}$ that provide oscillatory motions. Unlike the procedure considered by Elías-Zúñiga [11, 12], we do not assume an expression for the solution but solve the nonlinear differential equation exactly as was done in [15]. This is done using elliptic functions so that, after inversion, the solution $x$ is provided as an explicit function of time $t$. When $a_{1}=0$ and $a_{5}>0$, the system becomes a truly nonlinear oscillator [16] for which the exact expressions for the period and the solution have been already obtained [17]. The particular situation in which coefficients $a_{1}$ and $a_{5}$ are both positive is the most common case analysed. However, we obtain closedform exact solutions not only for the case in which both coefficients are positive, but also for all possible combinations of positive and negative values of these coefficients which provide periodic motions. The set of possible combinations of signs of these coefficients gives rise to four different cases. In three of these combinations ((a) $a_{1} \geq 0, a_{5}>0$, and $x_{0}>0$, (b) $a_{1}<0, a_{5}>0$ and $x_{0}>\left(-3 a_{1} / a_{5}\right)^{1 / 4}$, and (d) $a_{1}>0$, $a_{5}<0$ and $\left.0<x_{0}<\left(-a_{1} / a_{5}\right)^{1 / 4}\right)$ the system oscillates around the equilibrium position $x=0$ with $x \in\left[-x_{0}, x_{0}\right]$, where $x_{0}>0$ is the oscillation amplitude. However, there is still one more case $\left((\mathrm{c}) a_{1}>0, a_{5}<0\right.$, and $0<x_{0}<\left(-3 a_{1} / a_{5}\right)^{1 / 4}$ or $\left.-\left(-3 a_{1} / a_{5}\right)^{1 / 4}<x_{0}<0\right)$ in which the system does not oscillate around the position $x=0$ with $x \in\left[-x_{0}, x_{0}\right]$, but around the equilibrium position $x=\left(-a_{1} / a_{5}\right)^{1 / 4}$ with $x \in$ $\left[x_{1}, x_{0}\right]$. Three different sets of closed-form expressions for the exact period and solution were obtained. Following the procedure considered by Lai and Chow [18], some examples are analysed and plots including periods, solutions, or phasediagrams are presented and discussed.

\section{Formulation and Solution Procedure}

A quintic oscillator is an example of a conservative autonomous oscillatory system, which is modelled by the following second-order differential equation:

$$
\frac{d^{2} x}{d t^{2}}+a_{1} x+a_{5} x^{5}=0
$$

with initial conditions

$$
\begin{aligned}
x(0) & =x_{0}>0, \\
\frac{d x}{d t}(0) & =0 .
\end{aligned}
$$

In (1) $x$ and $t$ are generalized dimensionless displacement and time variables, and we assume that the coefficients for the linear and the nonlinear terms satisfy. In order to obtain the exact period and periodic solution for (1), we take into account that this is a conservative system and has the following first integral:

$$
\left(\frac{d x}{d t}\right)^{2}+a_{1} x^{2}+\frac{1}{3} a_{5} x^{6}=a_{1} x_{0}^{2}+\frac{1}{3} x_{0}^{6}
$$

which can be written as follows:

$$
v^{2}=\left(\frac{d x}{d t}\right)^{2}=a_{1}\left(x_{0}^{2}-x^{2}\right)+\frac{1}{3} a_{5}\left(x_{0}^{6}-x^{6}\right) \geq 0 .
$$

The dynamical study [19] of the nonlinear differential equation given in (1) showed that its motion is periodic in the following situations:

(a) $a_{1} \geq 0, a_{5}>0$, and $x_{0}>0$ : the system oscillates around the equilibrium position $x=0$ and the periodic solution $x$ satisfies $x \in\left[-x_{0}, x_{0}\right]$.

(b) $a_{1}<0, a_{5}>0$, and $x_{0}>\left(-3 a_{1} / a_{5}\right)^{1 / 4}$ : the system oscillates around the equilibrium position $x=0$ and the periodic solution $x$ satisfies $x \in\left[-x_{0}, x_{0}\right]$.

(c) $a_{1}<0, a_{5}>0$, and $0<x_{0}<\left(-3 a_{1} / a_{5}\right)^{1 / 4}$ : the system oscillates around the equilibrium position $x=\left(-3 a_{1} / a_{5}\right)^{1 / 4}$ and the periodic solution satisfies $x \in\left[x_{1}, x_{0}\right]$ with $0<x_{1} \leq x \leq x_{0}$ (or around the equilibrium position $x=-\left(-3 a_{1} / a_{5}\right)^{1 / 4}$ with $x \in$ $\left[x_{0}, x_{1}\right]$ when $\left.-\left(-3 a_{1} / a_{5}\right)^{1 / 4}<x_{0}<0\right)$.

(d) $a_{1}>0, a_{5}<0$, and $0<x_{0}<\left(-a_{1} / a_{5}\right)^{1 / 4}$ : the system oscillates around the equilibrium position $x=0$ and the periodic solution $x$ satisfies $x \in\left[-x_{0}, x_{0}\right]$.

The phase plots in Figure 1 illustrate four examples of these situations: (a) $a_{1}=1, a_{5}=3$, and $x_{0}=1$, (b) $a_{1}=-1$, $a_{5}=3$ with $x_{0}=1.1\left(x_{0}\right.$ must be $\left.>1\right)$, (c) $a_{1}=-1, a_{5}=-3$, and $x_{0}=0.95\left(x_{0}\right.$ must be $\left.<1\right)$, and (d) $a_{1}=1, a_{5}=-3$, 


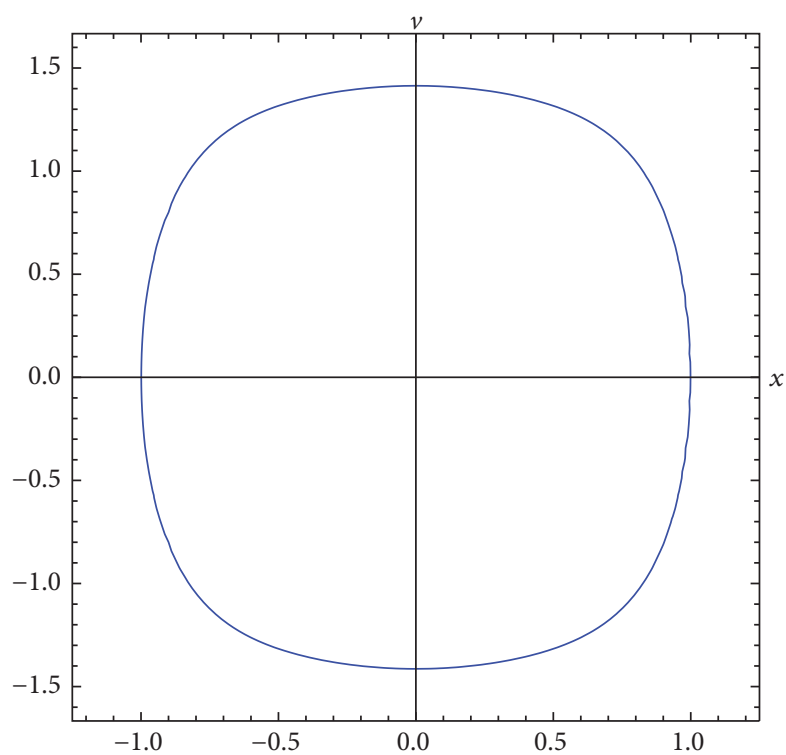

(a)

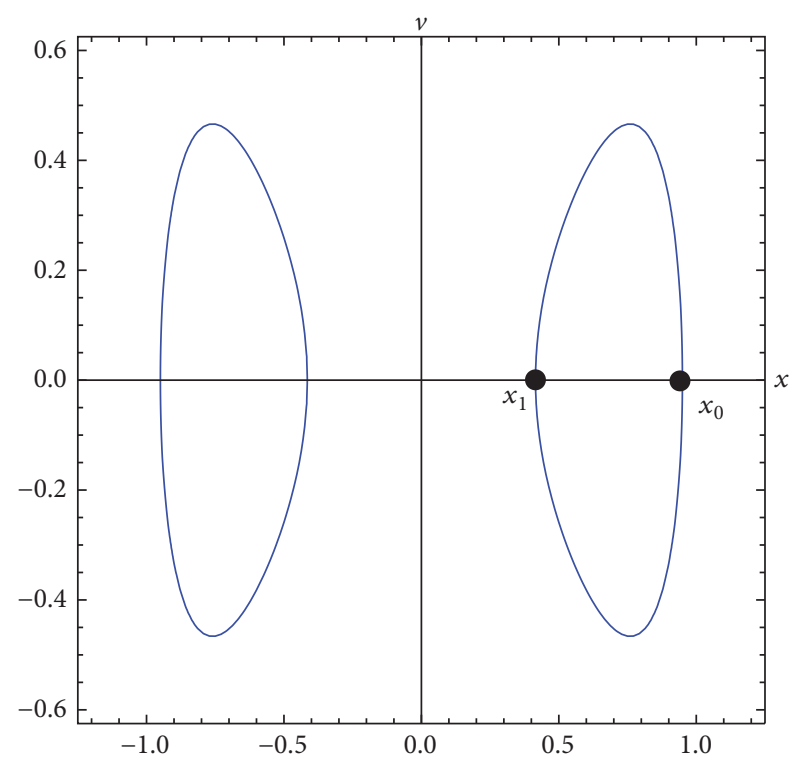

(c)

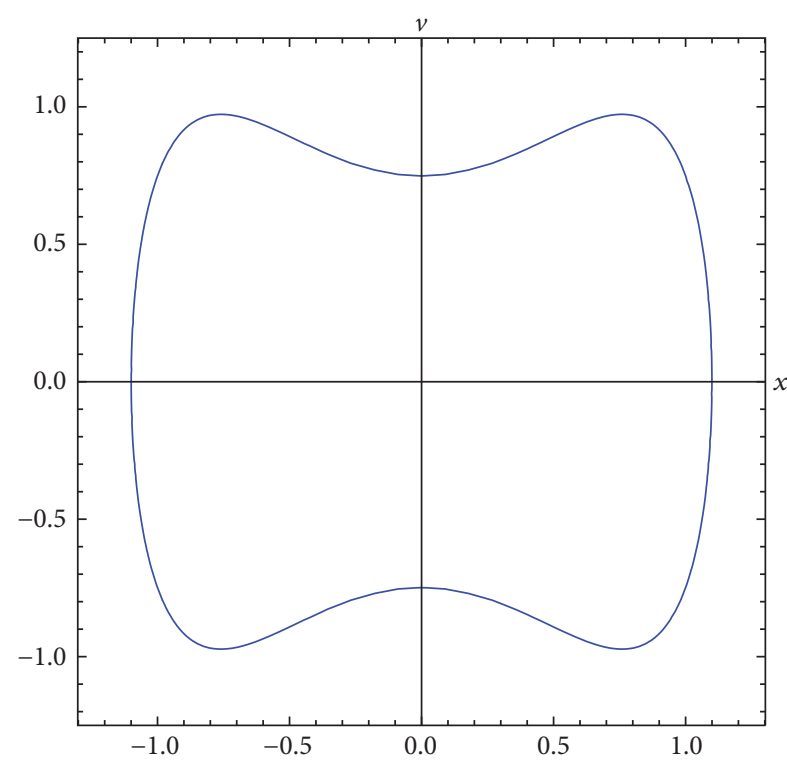

(b)

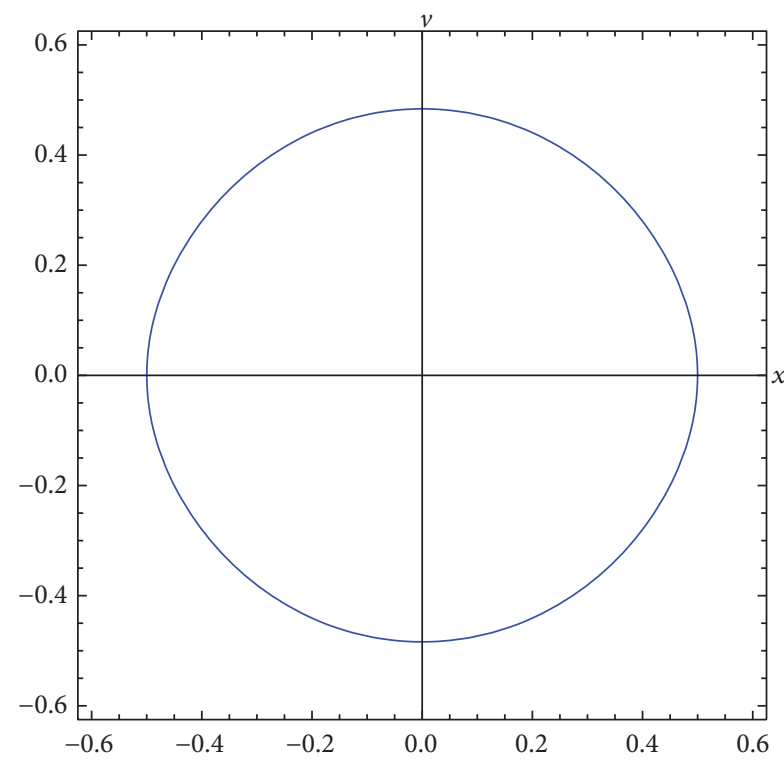

(d)

FIGURE 1: Phase space portraits for parameters values of (a) $a_{1}=1, a_{5}=3$, and $x_{0}=1$; (b) $a_{1}=-1, a_{5}=3$, and $x_{0}=1.1 ;$ (c) $a_{1}=-1, a_{5}=3$, and $x_{0}=0.95$; and (d) $a_{1}=1, a_{5}=-3$, and $x_{0}=0.5$.

and $x_{0}=0.5$ ( $x_{0}$ must be $\left.<3^{-1 / 4} \approx 0.7598\right)$. As can be seen in these figures, for cases (a), (b), and (d) the system oscillates around the equilibrium position $x=0$, whereas for case (c) the system oscillates around the equilibrium position $x=\left(-a_{1} / a_{5}\right)^{1 / 4}$. In the following sections we obtain the exact expressions for the period and the periodic solution for each of these four cases.

\section{Exact Solution When $a_{1} \geq 0, a_{5}>0$, and $x_{0}>0$}

In this situation all the solutions are periodical and the phase space diagram is made up of an infinite number of closed orbits, each of them for each value of the initial amplitude $x_{0}$ (as can be seen in Figure 1(a)). This system oscillates around the equilibrium position $x=0$ with $x \in\left[-x_{0}, x_{0}\right]$, where $x_{0}>0$ is the initial amplitude and the period, $T$, and periodic solution, $x$, are dependent on the oscillation amplitude $x_{0}$. This system corresponds to a nonlinear oscillator for which the nonlinear function $f(x)=a_{1} x+a_{3} x^{3}+a_{5} x^{5}$ is odd; that is, $f(-x)=-f(x)$. From (4) we obtain

$$
\left(\frac{d t}{d x}\right)^{2}=\frac{1}{a_{1}\left(x_{0}^{2}-x^{2}\right)+(1 / 3) a_{5}\left(x_{0}^{6}-x^{6}\right)}
$$


and then

$$
d t= \pm \frac{d x}{\sqrt{a_{1}\left(x_{0}^{2}-x^{2}\right)+(1 / 3) a_{5}\left(x_{0}^{6}-x^{6}\right)}}
$$

where the sign $( \pm)$ is chosen taking into account the sign of $d x / d t$ in each quadrant.

Taking into account that $a_{5}>0$, from (6) we can write

$$
\sqrt{\frac{a_{5}}{3}} d t= \pm \frac{d x}{\sqrt{\left(3 a_{1} / a_{5}\right)\left(x_{0}^{2}-x^{2}\right)+\left(x_{0}^{6}-x^{6}\right)}}
$$

and after some mathematical operations we obtain

$$
\sqrt{\frac{a_{5}}{3}} d t= \pm \frac{d x}{\sqrt{\left(x_{0}^{2}-x^{2}\right)\left(x^{4}+x_{0}^{2} x^{2}+3 a_{1} / a_{5}+x_{0}^{2}\right)}} .
$$

Integrating (8) we obtain

$$
\sqrt{\frac{a_{5}}{3}} t= \pm \int_{x}^{x_{0}} \frac{d x}{\sqrt{\left(x_{0}^{2}-x^{2}\right)\left(x^{4}+x_{0}^{2} x^{2}+3 a_{1} / a_{5}+x_{0}^{2}\right)}} .
$$

The change of variable $x^{2}=u$ gives

$$
\sqrt{\frac{a_{5}}{3}} t= \pm \int_{x^{2}}^{x_{0}^{2}} \frac{d u}{\sqrt{\left(x_{0}^{2}-u\right) u\left(u^{2}+x_{0}^{2} u+3 a_{1} / a_{5}+x_{0}^{2}\right)}} .
$$

This is an improper integral which contains a square root of a four-degree polynomial in the denominator and so its solution can be expressed as a function of an elliptic integral.

3.1. Calculation of the Exact Period. The symmetry of the problem indicates that the period of the oscillation $T$ is four times the time taken by the oscillator to go from $u=0$ to $u=x_{0}^{2}$. Therefore, from (10) it follows that

$$
T=2 \sqrt{\frac{3}{a_{5}}} \int_{x^{2}}^{x_{0}^{2}} \frac{d u}{\sqrt{\left(x_{0}^{2}-u\right) u\left(u^{2}+x_{0}^{2} u+3 a_{1} / a_{5}+x_{0}^{2}\right)}} .
$$

We consider the definite integral [20, section 3.145, formula 2, pages 270-271]

$$
\begin{aligned}
& \int_{\beta}^{y} \frac{d u}{\sqrt{(\alpha-u)(u-\beta)\left[(u-\sigma)^{2}+\rho^{2}\right]}}=\frac{1}{\sqrt{p q}} \\
& \cdot F\left(2 \operatorname{arccot} \sqrt{\frac{q(\alpha-y)}{p(y-\beta)}}, \frac{(\alpha-\beta)^{2}-(p-q)^{2}}{4 p q}\right),
\end{aligned}
$$

where $\beta<u<\alpha, F(\phi, m)$ is the incomplete elliptic integral of the first kind [20]

$$
F(\phi, m)=\int_{0}^{\phi} \frac{d \theta}{\sqrt{1-m \sin ^{2} \theta}},
$$

and $p, q$, and $m$ are defined as

$$
\begin{aligned}
& p=\sqrt{(\sigma-\alpha)^{2}+\rho^{2}}, \\
& q=\sqrt{(\sigma-\beta)^{2}+\rho^{2}}, \\
& m=\frac{(\alpha-\beta)^{2}-(p-q)^{2}}{4 p q} .
\end{aligned}
$$

By comparing the integrals in (11) and (12) we obtain $y=x_{0}^{2}$, $\alpha=x_{0}^{2}$, and $=0$, as well as the following values for the different parameters which appear in (12)

$$
\begin{aligned}
& \sigma=-\frac{1}{2} x_{0}^{2}, \\
& \rho=\frac{1}{2} \sqrt{\frac{12 a_{1}}{a_{5}}+3 x_{0}^{2}}, \\
& p=\sqrt{\frac{3}{a_{5}}} \sqrt{a_{1}+a_{5} x_{0}^{4}}, \\
& q=\frac{1}{\sqrt{a_{5}}} \sqrt{3 a_{1}+a_{5} x_{0}^{4}}, \\
& m=\frac{1}{2}-\frac{\sqrt{3}\left(2 a_{1}+a_{5} x_{0}^{4}\right)}{4 \sqrt{\left(a_{1}+a_{5} x_{0}^{4}\right)\left(3 a_{1}+a_{5} x_{0}^{4}\right)}} .
\end{aligned}
$$

As $y=\alpha=x_{0}^{2}$, then $F(2 \operatorname{arccot} 0, m)=F(\pi, m)=2 K(m)$, where $K(m)$ is the complete elliptic integral of the first kind defined as [20]

$$
K(m)=\int_{0}^{\pi / 2} \frac{d \theta}{\sqrt{1-m \sin ^{2} \theta}} .
$$

From (11) to (16) we conclude that the exact period of the quintic nonlinear oscillator can be written in the compact form

$$
T=4\left(\frac{3}{q_{1} q_{3}}\right)^{1 / 4} K\left(m_{a}\right)
$$

where $m_{a}$ takes the form

$$
m_{a}=\frac{1}{2}-\frac{q_{2}}{4}\left(\frac{3}{q_{1} q_{3}}\right)^{1 / 2}
$$

and coefficients $q_{n}$-which depend on $a_{1}, a_{5}$, and $x_{0}$-are defined as follows:

$$
q_{n}=n a_{1}+a_{5} x_{0}^{4} .
$$

Figure 2 shows the period $T$ as a function of the initial amplitude $x_{0}$ (17) when $a_{1}=1$ and (a) $a_{5}=0.1$, (b) $a_{5}=0.5$, (c) $a_{5}=5$, and (d) $a_{5}=50$. 


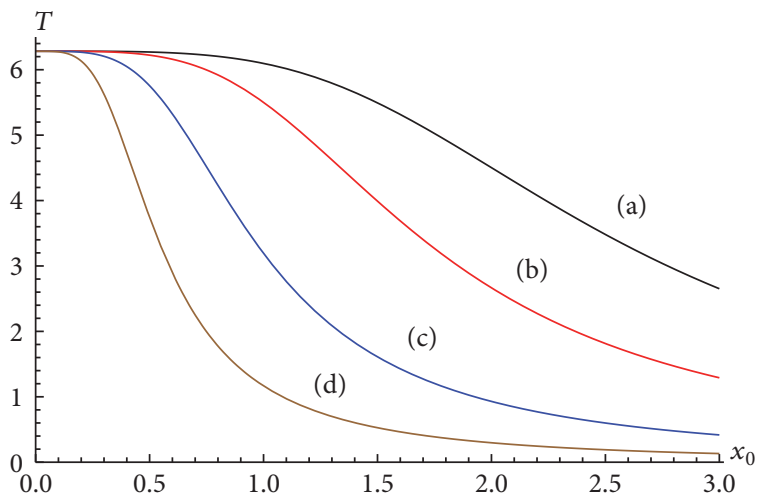

Figure 2: Exact period $T$ in (17) as a function of the initial amplitude $x_{0}$ when $a_{1}=1$ and (a) $a_{5}=0.1$, (b) $a_{5}=0.5$, (c) $a_{5}=5$, and (d) $a_{5}=50$.

3.2. Calculation of the Exact Solution. From (6) we obtain $t$ as a function of $x$ for the following cases:

Trajectory $1 \rightarrow 2\left(0 \leq t \leq T / 4\right.$ and $\left.x_{0} \geq x \geq 0\right), x$ is positive and $d x / d t$ is negative.

Trajectory $2 \rightarrow 3\left(T / 4 \leq t \leq T / 2\right.$ and $\left.0 \geq x \geq-x_{0}\right)$, $x$ is negative and $d x / d t$ is negative.

Trajectory $3 \rightarrow 4\left(T / 2 \leq t \leq 3 T / 4\right.$ and $\left.-x_{0} \leq x \leq 0\right)$, $x$ is negative and $d x / d t$ is positive.

Trajectory $4 \rightarrow 1\left(3 T / 4 \leq t \leq T\right.$ and $\left.0 \leq x \leq x_{0}\right), x$ is positive and $d x / d t$ is positive.

From (10), it follows that for trajectory $1 \rightarrow 2(0 \leq t \leq T / 4$ and $x_{0} \geq x \geq 0$ ) we have

$$
2 \sqrt{\frac{a_{5}}{3}} t=\int_{x^{2}}^{x_{0}^{2}} \frac{d u}{\sqrt{\left(x_{0}^{2}-u\right) u\left(u^{2}+x_{0}^{2} u+3 a_{1} / a_{5}+x_{0}^{2}\right)}}
$$

The definite integral in (20) can be split as follows:

$$
\begin{aligned}
& 2 \sqrt{\frac{a_{5}}{3}} t \\
& =\int_{0}^{x_{0}^{2}} \frac{d u}{\sqrt{\left(x_{0}^{2}-u\right) u\left(u^{2}+x_{0}^{2} u+3 a_{1} / a_{5}+x_{0}^{2}\right)}} \\
& \quad-\int_{0}^{x^{2}} \frac{d u}{\sqrt{\left(x_{0}^{2}-u\right) u\left(u^{2}+x_{0}^{2} u+3 a_{1} / a_{5}+x_{0}^{2}\right)}} .
\end{aligned}
$$

The values of the two integrals on the right-hand side of (21) can be calculated taking into account (11) and (12) and their values are

$$
\begin{aligned}
& \int_{0}^{x_{0}^{2}} \frac{d u}{\sqrt{\left(x_{0}^{2}-u\right) u\left(u^{2}+x_{0}^{2} u+3 a_{1} / a_{5}+x_{0}^{2}\right)}} \\
& \quad=2 \sqrt{\frac{a_{5}}{3} \frac{T}{4}}, \\
& \int_{0}^{x^{2}} \frac{d u}{\sqrt{\left(x_{0}^{2}-u\right) u\left(u^{2}+x_{0}^{2} u+3 a_{1} / a_{5}+x_{0}^{2}\right)}} \\
& \quad=\frac{1}{\sqrt{p q}} F\left(2 \operatorname{arccot} \sqrt{\frac{q\left(A^{2}-x^{2}\right)}{p x^{2}}}, m_{a}\right) .
\end{aligned}
$$

Substituting (22) into (21) gives

$$
\begin{aligned}
& 2 \sqrt{p q} \sqrt{\frac{a_{5}}{3}}\left(\frac{T}{4}-t\right) \\
& \quad=F\left(2 \operatorname{arccot} \sqrt{\frac{q\left(A^{2}-x^{2}\right)}{p x^{2}}}, m_{a}\right),
\end{aligned}
$$

and using (15)-(19), (23) can be written as

$$
\begin{aligned}
& 2 K\left(m_{a}\right)-\left(\frac{q_{1} q_{3}}{3}\right)^{1 / 4} t \\
& \quad=F\left(2 \operatorname{arccot}\left[\left(\frac{q_{3}}{3 q_{1}}\right)^{1 / 4}\left(\frac{x_{0}^{2}-x^{2}}{x^{2}}\right)^{1 / 2}\right], m_{a}\right) .
\end{aligned}
$$

The inverse function of $F(\varphi, m)$ is the Jacobi amplitude $\varphi$ [21, 22]

$$
F^{-1}(u, m)=\varphi=\operatorname{am}(u, m)
$$

whose cosine is the Jacobi cosine function, $\mathrm{cn}[21]$

$$
\cos \varphi=\cos (\operatorname{am}(u, m))=\operatorname{cn}(u, m) .
$$

In order to introduce an "arccos" function in (24) we take into account that

$$
2 \operatorname{arccot} z=\varphi \text {, }
$$

where

$$
z=\left(\frac{q_{3}}{3 q_{1}}\right)^{1 / 4}\left(\frac{x_{0}^{2}-x^{2}}{x^{2}}\right)^{1 / 2}
$$

From (28) we obtain

$$
\tan ^{2} \frac{\varphi}{2}=\frac{1}{z^{2}}
$$


Taking into account the relation

$$
\tan ^{2} \frac{\varphi}{2}=\frac{1-\cos \varphi}{1+\cos \varphi}
$$

we can finally write

$$
\cos \varphi=\frac{z^{2}-1}{z^{2}+1}=\frac{\sqrt{q_{3}}\left(x_{0}^{2}-x^{2}\right)-\sqrt{3 q_{1}} x^{2}}{\sqrt{q_{3}}\left(x_{0}^{2}-x^{2}\right)+\sqrt{3 q_{1}} x^{2}}
$$

which allows us to write (24) as follows:

$$
\begin{aligned}
2 K & \left(m_{a}\right)-2\left(\frac{q_{1} q_{3}}{3}\right)^{1 / 4} t \\
& =F\left(\arccos \left(\frac{\sqrt{q_{3}}\left(x_{0}^{2}-x^{2}\right)-\sqrt{3 q_{1}} x^{2}}{\sqrt{q_{3}}\left(x_{0}^{2}-x^{2}\right)+\sqrt{3 q_{1}} x^{2}}\right), m_{a}\right)
\end{aligned}
$$

and from (25), (26), and (32) we can write, after some simplifications,

$$
\begin{aligned}
& \frac{\sqrt{q_{3}} x_{0}^{2}-\left(\sqrt{q_{3}}+\sqrt{3 q_{1}}\right) x^{2}}{\sqrt{q_{3}} x_{0}^{2}-\left(\sqrt{q_{3}}-\sqrt{3 q_{1}}\right) x^{2}} \\
& =\operatorname{cn}\left(2 K\left(m_{a}\right)-2\left(\frac{1}{3} q_{1} q_{3}\right)^{1 / 4} t, m_{a}\right) \\
& =-\operatorname{cn}\left(2\left(\frac{1}{3} q_{1} q_{3}\right)^{1 / 4} t, m_{a}\right),
\end{aligned}
$$

$$
\left.+\sqrt{\frac{3 q_{1}}{q_{3}}}\left(\frac{1-\operatorname{cn}\left((1 / 2)\left((8 / 3) q_{1} q_{3}\right)^{1 / 4} t, m_{a}\right)}{1+\operatorname{cn}\left((1 / 2)\left((8 / 3) q_{1} q_{2}\right)^{1 / 4} t, m_{a}\right)}\right)\right]^{-1 / 2}
$$

which is valid for $0 \leq t \leq T / 4$.

From (7), it follows for trajectory $2 \rightarrow 3$ that

$$
\begin{aligned}
& 2 \sqrt{\frac{a_{5}}{3}} \int_{T / 4}^{t} d t=2 \sqrt{\frac{a_{5}}{3}}\left(t-\frac{T}{4}\right) \\
& =-\int_{0}^{x^{2}} \frac{d u}{\sqrt{\left(x_{0}^{2}-u\right) u\left(u^{2}+x_{0}^{2} u+3 a_{1} / a_{5}+x_{0}^{2}\right)}}
\end{aligned}
$$

and for trajectory $3 \rightarrow 4(T / 4 \leq t \leq T / 2)$

$$
\begin{gathered}
2 \sqrt{\frac{a_{5}}{3}} \int_{T / 2}^{t} d t=2 \sqrt{\frac{a_{5}}{3}}\left(t-\frac{T}{2}\right) \\
=\int_{x_{0}^{2}}^{x^{2}} \frac{d u}{\sqrt{\left(x_{0}^{2}-u\right) u\left(u^{2}+x_{0}^{2} u+3 a_{1} / a_{5}+x_{0}^{2}\right)}} .
\end{gathered}
$$

Proceeding in the same manner as for trajectory $1 \rightarrow 2$, it is follows that $x_{b}(t)=-x_{a}(t)$ which is valid for $T / 4 \leq t \leq 3 T / 4$, because $x<0$ for these values of time.

Finally, for trajectory $4 \rightarrow 1$ we have

$$
\begin{aligned}
2 & \sqrt{\frac{a_{5}}{3}} \int_{3 T / 4}^{t} d t=2 \sqrt{\frac{a_{5}}{3}}\left(t-\frac{3 T}{4}\right) \\
& =\int_{0}^{x^{2}} \frac{d u}{\sqrt{\left(x_{0}^{2}-u\right) u\left(u^{2}+x_{0}^{2} u+3 a_{1} / a_{5}+x_{0}^{2}\right)}}
\end{aligned}
$$

and we obtain the same value for the solution as that given in (34).

The exact solution of the quintic oscillator can be written as follows:

$$
x(t)= \begin{cases}x_{a}(t) & 0 \leq t \leq \frac{T}{4} \\ -x_{a}(t) & \frac{T}{4} \leq t \leq \frac{3 T}{4} \\ x_{a}(t) & \frac{3 T}{4} \leq t \leq T\end{cases}
$$

Taking into account the relation [22, formula 16.18.4, page 574]

$$
\frac{1-\operatorname{cn} 2 u}{1+\operatorname{cn} 2 u}=\frac{\operatorname{sn}^{2} u d n^{2} u}{\operatorname{cn}^{2} u}
$$

and (34) and (38), the exact periodic solution of the quintic oscillator can be written in compact form as follows:

$$
x(t)=\frac{x_{0} \operatorname{cn}\left(\left((1 / 3) q_{1} q_{3}\right)^{1 / 4} t, m_{a}\right)}{\left[\operatorname{cn}^{2}\left(\left((1 / 3) q_{1} q_{3}\right)^{1 / 4} t, m_{a}\right)+\left(3 q_{1} / q_{3}\right)^{1 / 2} \operatorname{sn}^{2}\left(\left((1 / 3) q_{1} q_{3}\right)^{1 / 4} t, m_{a}\right) \operatorname{dn}^{2}\left(\left((1 / 3) q_{1} q_{3}\right)^{1 / 4} t, m_{a}\right)\right]^{1 / 2}}
$$




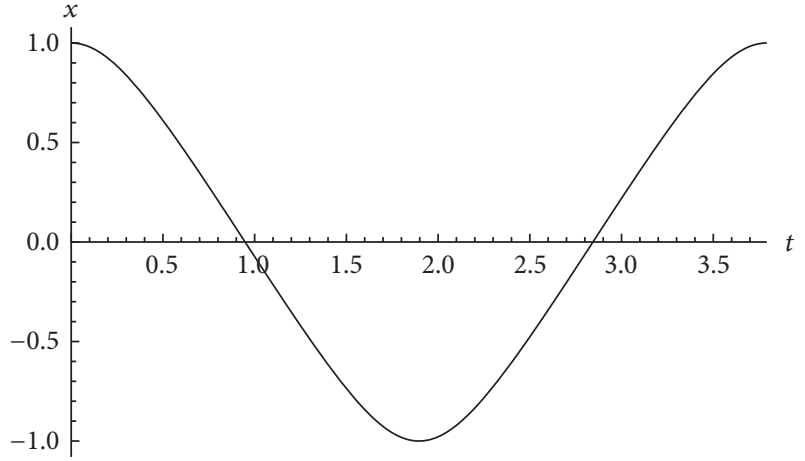

Figure 3: Exact solution $x$ in (40) as a function of time $t$ when $a_{1}=$ $1, a_{5}=3$, and $x_{0}=1$.

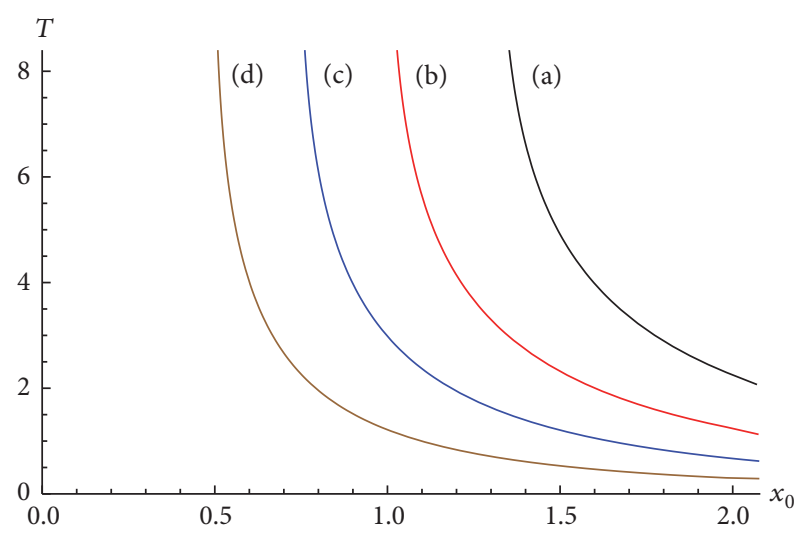

FIGURE 4: Exact period $T$ in (17) as a function of the initial amplitude $x_{0}$ when $a_{1}=-1, x_{0}>\left(-3 a_{1} / a_{5}\right)^{1 / 4}$ and (a) $a_{5}=1$, (b) $a_{5}=3$, (c) $a_{5}=10$, and (d) $a_{5}=50$.

which is valid for all value of $t$ and where $\mathrm{cn}, \mathrm{sn}$, and $\mathrm{dn}$ are the basic Jacobi elliptic functions [20]. Figure 3 shows the plot of the displacement $x$ as a function of time $t$ when $a_{1}=1, a_{5}=$ 3 , and $x_{0}=1$. This displacement was obtained using (40). This figure corresponds to the phase space portrait shown in Figure 1(a).

\section{Exact Solution When $a_{1}<0, a_{5}>0$, and $x_{0}>$} $\left(-3 a_{1} / a_{5}\right)^{1 / 4}$

Equations (17) and (40) can be used to obtain the period and the solution in case (b), $a_{1}<0, a_{5}>0$, and $x_{0}>$ $\left(-3 a_{1} / a_{5}\right)^{1 / 4}$. Figure 4 shows the period $T$ as a function of the initial amplitude $x_{0}$ (17) when $a_{1}=-1$ and (a) $a_{5}=1$, (b) $a_{5}=3$, (c) $a_{5}=10$, and (d) $a_{5}=50$. Figure 5 shows the plot of the displacement $x$ as a function of time $t$ when $a_{1}=-1, a_{5}=3$, and $x_{0}=1.1$. This displacement was obtained using (40). This figure corresponds to the phase space portrait shown in Figure 1(b).

\section{Exact Solution When $a_{1}<0, a_{5}>0$, and $0<x_{0}<\left(-3 a_{1} / a_{5}\right)^{1 / 4}$}

The phase portrait in Figure 1(c) shows the behaviour of the oscillator when $0<x_{0}<\left(-3 a_{1} / a_{5}\right)^{1 / 4}\left(a_{1}=-1, a_{5}=3\right.$, and

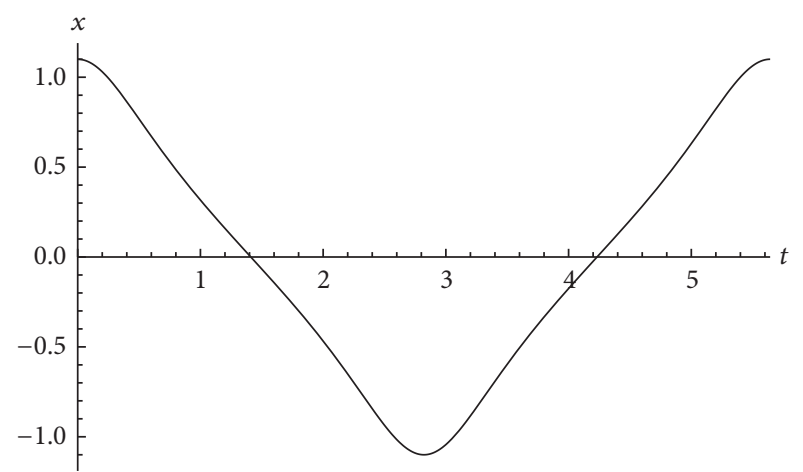

FIgURE 5: Exact solution $x$ in (40) as a function of time $t$ when $a_{1}=$ $-1, a_{5}=3$, and $x_{0}=1.1$.

$\left.x_{0}=19 / 20=0.95<1=\left(-3 a_{1} / a_{5}\right)^{1 / 4}\right)$. As was previously mentioned, now the system oscillates around the equilibrium position $x=\left(-a_{1} / a_{5}\right)^{1 / 4}$ and the periodic solution satisfies $x \in\left[x_{1}, x_{0}\right]$ with $0<x_{1} \leq x \leq x_{0}$.

5.1. Calculation of the Exact Period. We shall now obtain the period and the solution for the right orbit in Figure 1(c) for which $x>0$ taking into account that $x_{0}$ is the highest value for $x$. From (7) and considering that $x>0$, we obtain $t$ as a function of $x$ as follows:

$$
2 \sqrt{\frac{a_{5}}{3}} t=\int_{x^{2}}^{x_{0}^{2}} \frac{d u}{\sqrt{\left(x_{0}^{2}-u\right) u\left(u^{2}+x_{0}^{2} u+3 a_{1} / a_{5}+x_{0}^{2}\right)}}
$$

which can be written as

$$
2 \sqrt{\frac{a_{5}}{3}} t=\int_{x^{2}}^{x_{0}^{2}} \frac{d u}{\sqrt{\left(x_{0}^{2}-u\right)\left(u-u_{1}\right) u\left(u-u_{2}\right)}}
$$

where $u_{1}$ and $u_{2}$ are defined by the following equations:

$$
\begin{aligned}
u_{1} & =\frac{1}{2 \sqrt{a_{5}}}\left(\sqrt{-3\left(4 a_{1}+a_{5} x_{0}^{4}\right)}-\sqrt{a_{5} x_{0}^{4}}\right) \\
& =\frac{1}{2 \sqrt{a_{5}}}\left(\sqrt{-3 q_{4}}-\sqrt{q_{0}}\right), \\
u_{2} & =-\frac{1}{2 \sqrt{a_{5}}}\left(\sqrt{-3\left(4 a_{1}+a_{5} x_{0}^{4}\right)}+\sqrt{a_{5} x_{0}^{4}}\right) \\
& =-\frac{1}{2 \sqrt{a_{5}}}\left(\sqrt{-3 q_{4}}+\sqrt{q_{0}}\right),
\end{aligned}
$$

where $q_{2}$ and $q_{4}$ are defined in (19).

For the right orbit in Figure 1(c) it is easy to verify that it satisfies

$$
x_{0}^{2} \geq u \geq u_{1}>0>u_{2}
$$


Now the period, $T$, and the periodic solution, $x$, are dependent on $x_{0}$ and $x \in\left[x_{1}, x_{0}\right]$, where $x_{1}=\sqrt{u_{1}}$ and $u_{1}$ is given in (43). The following integral is valid for $a \geq u \geq b>c>d$ $\left[20,6^{\text {th }}\right.$ edition, Formula 3.147, Integral 7, page 272]:

$$
\begin{aligned}
\int_{u}^{a} & \frac{d y}{\sqrt{(a-y)(y-b)(y-c)(y-d)}} \\
& =\frac{2}{\sqrt{(a-c)(b-d)}} F(\mu, r),
\end{aligned}
$$

where $F(\mu, r)$ is the incomplete elliptic integral of the first kind defined in (13) and $\mu$ and $r$ are given by the following equations:

$$
\begin{aligned}
& \mu=\arcsin \sqrt{\frac{(b-d)(a-u)}{(a-b)(u-d)}}, \\
& r=\frac{(a-b)(c-d)}{(a-c)(b-d)} .
\end{aligned}
$$

We have $a=x_{0}^{2}, b=u_{1}, c=0$, and $d=u_{2}$.

Then the value of the integral in (42) is

$$
\begin{aligned}
& 2 \sqrt{\frac{a_{5}}{3}} t=\frac{2}{\sqrt{x_{0}^{2}\left(u_{1}-u_{2}\right)}} \\
& \quad \cdot F\left(\arcsin \sqrt{\frac{\left(u_{1}-u_{2}\right)\left(x_{0}^{2}-x^{2}\right)}{\left(x_{0}^{2}-u_{1}\right)\left(x^{2}-u_{2}\right)}}, \frac{u_{2}\left(u_{1}-x_{0}^{2}\right)}{x_{0}^{2}\left(u_{1}-u_{2}\right)}\right) .
\end{aligned}
$$

As can be seen in Figure 1(c), the period of the oscillation is twice the time taken by the oscillator to go from $u=x_{0}^{2}$ to $u=u_{1}$. Therefore,

$$
T=2\left(\frac{-3}{q_{0} q_{4}}\right)^{1 / 4} K\left(m_{c}\right)
$$

where $m_{c}$ is given by

$$
m_{c}=\frac{1}{2}+\frac{q_{2}}{2}\left(\frac{-3}{q_{0} q_{4}}\right)^{1 / 2}
$$

$q_{0}, q_{2}$, and $q_{4}$ are defined in (19) and $K(m)$ is the complete elliptic integral of the first kind defined in (16). Figure 6 shows the period $T$ as a function of the initial amplitude $x_{0}$ (49) when $a_{1}=-1,0<x_{0}<\left(-3 a_{1} / a_{5}\right)^{1 / 4}$ and (a) $a_{5}=1$, (b) $a_{5}=3$, (c) $a_{5}=10$, and (d) $a_{5}=50$. This figure corresponds to the right orbit in the phase space portrait shown in Figure 1(c). Figure 7 shows the variation in the period as a function of the initial position when $a_{1}=-1$ and $a_{5}=3$, (a) $x_{0}>\left(-3 a_{1} / a_{5}\right)^{1 / 4}=1$, Section 4 (17), and (b) $0<x_{0}<\left(-3 a_{1} / a_{5}\right)^{1 / 4}=1$, Section 3 (49). As can be seen the motion is not periodic when the initial position is $x=\left(-3 a_{1} / a_{5}\right)^{1 / 4}$

5.2. Calculation of the Exact Solution. In order to obtain the solution $x$ as a function of time $t$, from (48) it follows that

$$
\begin{aligned}
& \frac{\left(u_{1}-u_{2}\right)\left(x_{0}^{2}-x^{2}\right)}{\left(x_{0}^{2}-u_{1}\right)\left(x^{2}-u_{2}\right)} \\
& \quad=\operatorname{sn}^{2}\left(\sqrt{\frac{1}{3} a_{5} x_{0}^{2}\left(u_{1}-u_{2}\right)} t, \frac{u_{2}\left(u_{1}-x_{0}^{2}\right)}{x_{0}^{2}\left(u_{1}-u_{2}\right)}\right)
\end{aligned}
$$

and taking into account (19), (43), (44), and (50), (51) allows us to obtain the solution as follows:

$$
x(t)=x_{0}\left[\frac{1-\left(1 / 2+\left(q_{2} / 2\right)\left(-3 / q_{0} q_{4}\right)^{1 / 2}\right) \operatorname{sn}^{2}\left(\left(-(1 / 3) q_{0} q_{4}\right)^{1 / 4} t, m_{c}\right)}{1-\left(1 / 2-(1 / 2)\left(-3 q_{0} / q_{4}\right)^{1 / 2}\right) \operatorname{sn}^{2}\left(\left(-(1 / 3) q_{0} q_{4}\right)^{1 / 4} t, m_{c}\right)}\right]^{1 / 2} .
$$

When $a_{1}<0$ and $a_{5}>0$, (49) and (52) are valid provided that the initial position $x_{0}$ satisfies the condition $0<x_{0}<$ $\left(-3 a_{1} / a_{5}\right)^{1 / 4}$, except for the equilibrium point (when $x_{0}=$ $\left.\left(-a_{1} / a_{5}\right)^{1 / 4}\right)$.

The expressions for the period and the exact solution for the left orbit in Figure 1(c), that is, for $x<0$, can be obtained following the same procedure as that used for $x>0$ and are not included here.

Figure 8(a) shows the plot of the displacement $x$ as a function of time $t$ when $a_{1}=-1, a_{5}=3$, and $x_{0}=19 / 20=0.95$.
This displacement was obtained using (52). In this example, and from (43), we obtain

$$
x_{1}=\sqrt{u_{1}}=\frac{1}{20} \sqrt{\frac{1}{2}(-361+\sqrt{249037})} \approx 0.415385 .
$$

As can easily be verified in Figure 8(a), the oscillatory motion of this system is bounded between $x_{1} \approx 0.415385$ and 


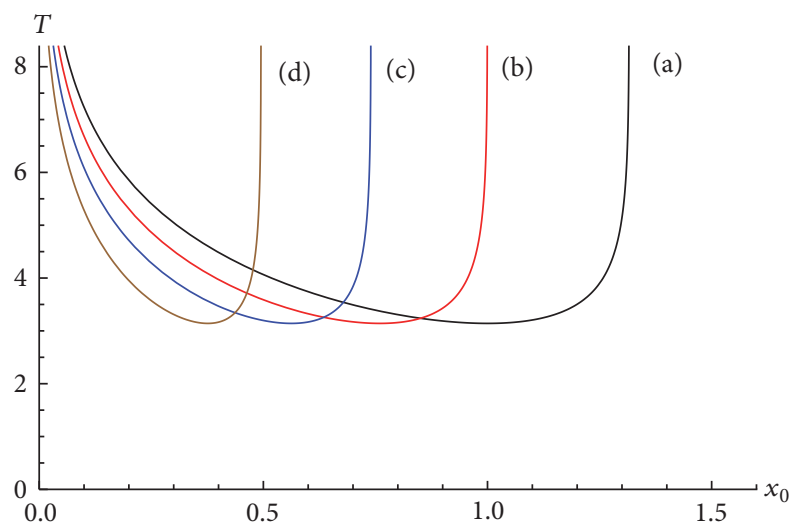

Figure 6: Exact period $T$ in (49) as a function of the initial amplitude $x_{0}$ when $a_{1}=-1,0<x_{0}<\left(-3 a_{1} / a_{5}\right)^{1 / 4}$ and (a) $a_{5}=1$, (b) $a_{5}=3$, (c) $a_{5}=10$, and (d) $a_{5}=50$.

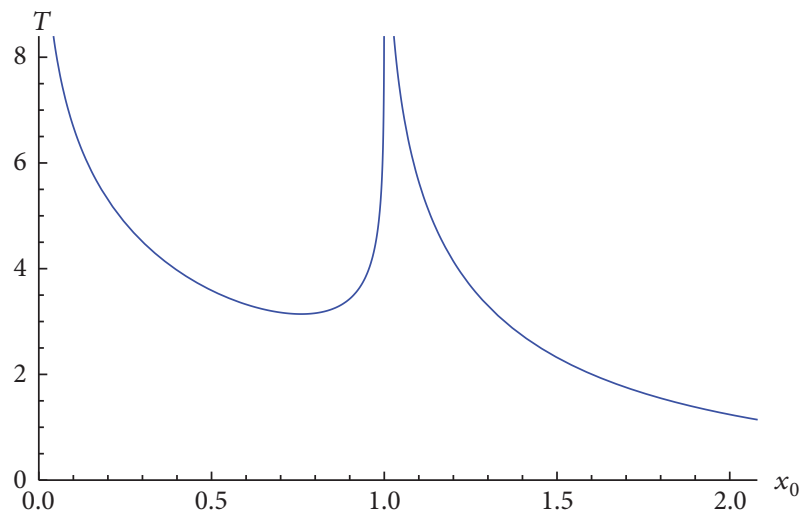

Figure 7: Exact period $T$ as a function of the initial position $x_{0}$ when $a_{1}=-1$ and $a_{5}=3$, (a) $x_{0}>\left(-3 a_{1} / a_{5}\right)^{1 / 4}=1$, Section 4 (17), and (b) $x_{0}<\left(-3 a_{1} / a_{5}\right)^{1 / 4}=1$, Section 3 (49). As can be seen the motion is not periodic when the initial position is $x=\left(-3 a_{1} / a_{5}\right)^{1 / 4}$.

$x_{0}=0.95$ and the equilibrium point is located at $x=\left(-a_{1} /\right.$ $\left.a_{5}\right)^{1 / 4}=3^{-1 / 4} \approx 0.759839$. Figure $8(\mathrm{~b})$ shows the displacement $x$ as a function of time $t$ when $a_{1}=-1, a_{5}=3$, and

$$
x_{0}=\frac{1}{20} \sqrt{\frac{1}{2}(-361+\sqrt{249037})} \approx 0.415385 .
$$

In this situation we obtained $x_{1}=\sqrt{u_{1}}=19 / 20=0.95$.

It is easy to verify that in this case the system is equivalent to a mixed-parity nonlinear oscillator. The change of variable

$$
y=x-\left(-\frac{a_{1}}{a_{5}}\right)^{1 / 4}
$$

gives

$$
\begin{aligned}
\frac{d^{2} y}{d t^{2}}- & 4 a_{1} y-10\left(-a_{1} a_{5}^{1 / 3}\right)^{3 / 4} y^{2}+10\left(-a_{1} a_{5}\right)^{1 / 2} y^{3} \\
& -5\left(-a_{1} a_{5}^{1 / 3}\right)^{1 / 4} y^{4}+a_{5} y^{5}=0 .
\end{aligned}
$$

The system modelled by (56) oscillates around the equilibrium position $y=0$ and the periodic solution $y$ satisfies $y \in\left[y_{1}, y_{0}\right]$ with $y_{1}=x_{1}-\left(-a_{1} / a_{5}\right)^{1 / 4}$ and $y_{0}=x_{0}-$ $\left(-a_{1} / a_{5}\right)^{1 / 4}$. Figure 9 shows the phase portrait when $a_{1}=-1$, $a_{5}=3$, and $y_{0}=x_{0}-\left(-a_{1} / a_{5}\right)^{1 / 4}=0.95-3^{-1 / 4} \approx 0.190164$. As can be seen the system oscillates around the equilibrium point $y=0$.

\section{Exact Solution When $a_{1}>0, a_{5}<0$, and $0<x_{0}<\left(-a_{1} / a_{5}\right)^{1 / 4}$}

For case (d) it is necessary to obtain the equation for the period again since if $a_{5}<0$, the root $\sqrt{a_{5} / 3}$ is not a real number and so (7) cannot be used. In order to obtain the exact solution when $a_{1}>0, a_{5}<0$, and $0<x_{0}<\left(-a_{1} / a_{5}\right)^{1 / 4}$ in which case the system oscillates around the equilibrium position $x=0$ and the periodic solution $x$ satisfies $x \in$ $\left[-x_{0}, x_{0}\right]$, we proceed as follows. From (6) we can write

$$
\sqrt{-\frac{a_{5}}{3}} d t= \pm \frac{d x}{\sqrt{\left(-3 a_{1} / a_{5}\right)\left(x_{0}^{2}-x^{2}\right)+\left(x_{0}^{6}-x^{6}\right)}} .
$$

6.1. Calculation of the Exact Period. We can consider the same four trajectories that we analysed at the beginning of Section 3.2. Doing the change of variable $x^{2}=u$, it follows that for trajectory $1 \rightarrow 2\left(0 \leq t \leq T / 4\right.$ and $\left.x_{0} \geq x \geq 0\right)$ we have

$$
\begin{aligned}
& 2 \sqrt{-\frac{a_{5}}{3}} t \\
& =\int_{x^{2}}^{x_{0}^{2}} \frac{d u}{\sqrt{\left(x_{0}^{2}-u\right) u\left(-u^{2}-x_{0}^{2} u-3 a_{1} / a_{5}-x_{0}^{2}\right)}}
\end{aligned}
$$

which can be written as

$$
2 \sqrt{-\frac{a_{5}}{3}} t=\int_{x^{2}}^{x_{0}^{2}} \frac{d u}{\sqrt{\left(w_{1}-u\right)\left(x_{0}^{2}-u\right) u\left(u-w_{2}\right)}},
$$

where $w_{1}$ and $w_{2}$ are defined by the following equations:

$$
\begin{aligned}
w_{1} & =\frac{1}{2 \sqrt{-a_{5}}}\left(\sqrt{3\left(4 a_{1}+a_{5} x_{0}^{4}\right)}-\sqrt{-a_{5} x_{0}^{4}}\right) \\
& =\frac{1}{2 \sqrt{-a_{5}}}\left(\sqrt{3 q_{4}}-\sqrt{-q_{0}}\right), \\
w_{2} & =-\frac{1}{2 \sqrt{-a_{5}}}\left(\sqrt{3\left(4 a_{1}+a_{5} x_{0}^{4}\right)}+\sqrt{-a_{5} x_{0}^{4}}\right) \\
& =-\frac{1}{2 \sqrt{a_{5}}}\left(\sqrt{3 q_{4}}+\sqrt{-q_{0}}\right) .
\end{aligned}
$$

It is easy to verify that it satisfies

$$
w_{1}>x_{0}^{2} \geq u>0>w_{2} .
$$

Now the period, $T$, and the periodic solution, $x$, are dependent on $x_{0}$ and $x \in\left[-x_{0}, x_{0}\right]$. The following integral is valid 


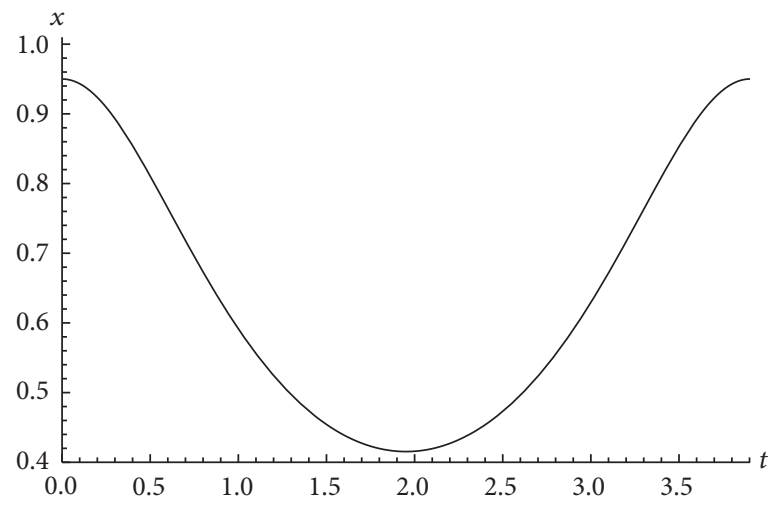

(a)

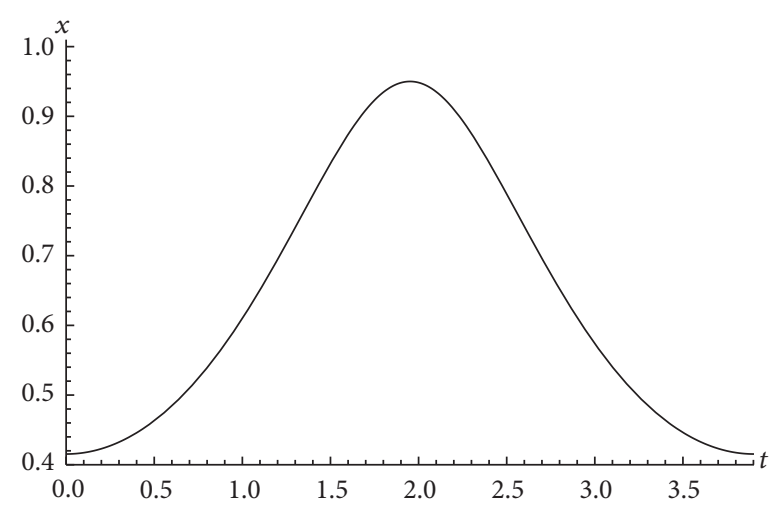

(b)

Figure 8: (a) Exact periodic solution $x$ in (52) for $a_{1}=-1, a_{5}=3$, and $x_{0}=19 / 20=0.95$ and (b) $a_{1}=-1, a_{5}=3$, and $x_{0}=(1 / 20) \sqrt{(1 / 2)(-361+\sqrt{249037})}$. In this situation $x_{1}=19 / 20=0.95$ is obtained.

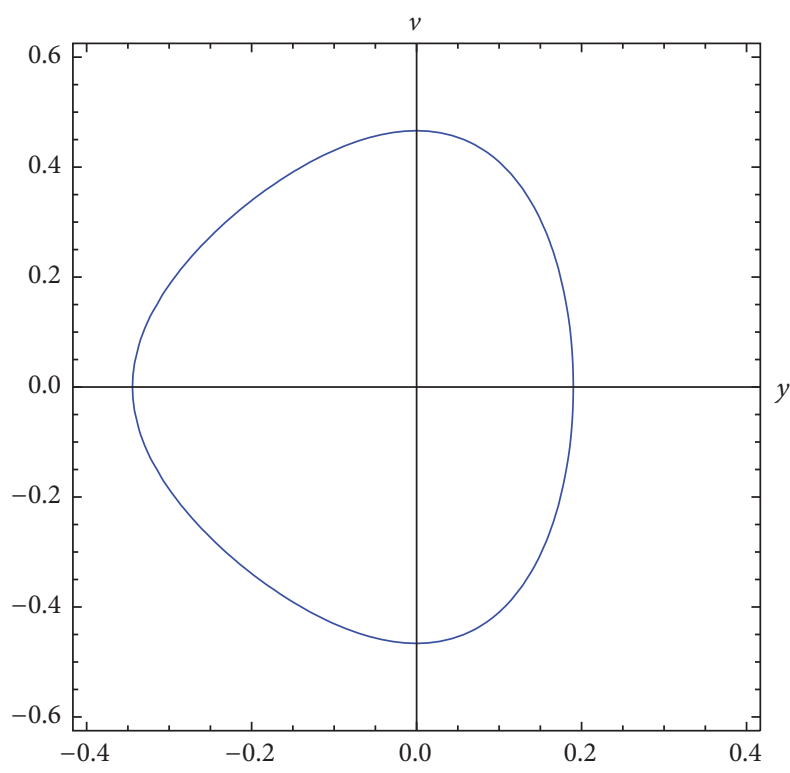

FIGURE 9: Phase space portrait for the mixed-parity nonlinear oscillator in (56) when $a_{1}=-1, a_{1}=3$ and $y_{0}=x_{0}-\left(-a_{1} / a_{5}\right)^{1 / 4}=$ $0.95-3^{-1 / 4} \approx 0.190164$. As can be seen the system oscillates around the equilibrium point $y=0$.

for $a>b \geq u>c>d\left[20,6^{\text {th }}\right.$ edition, Formula 3.147, Integral 5, page 272]:

$$
\begin{aligned}
& \int_{u}^{b} \frac{d y}{\sqrt{(a-y)(b-y)(y-c)(y-d)}} \\
& \quad=\frac{2}{\sqrt{(a-c)(b-d)}} F(\kappa, q),
\end{aligned}
$$

where $F(\kappa, q)$ is the incomplete elliptic integral of the first kind defined in (13) and $\kappa$ and $q$ are given by the following equations:

$$
\kappa=\arcsin \sqrt{\frac{(a-c)(b-u)}{(b-c)(a-u)}},
$$

$$
q=\frac{(b-c)(a-d)}{(a-c)(b-d)}
$$

We have $a=w_{1}, b=x_{0}^{2}, c=0$, and $d=w_{2}$. Then the value of the integral in (59) is

$$
\begin{gathered}
2 \sqrt{-\frac{a_{5}}{3}} t=\frac{2}{\sqrt{w_{1}\left(x_{0}^{2}-w_{2}\right)}} \\
\cdot F\left(\arcsin \sqrt{\frac{w_{1}\left(x_{0}^{2}-x^{2}\right)}{x_{0}^{2}\left(w_{1}-x^{2}\right)}}, \frac{x_{0}^{2}\left(w_{1}-w_{2}\right)}{w_{1}\left(x_{0}^{2}-w_{2}\right)}\right) .
\end{gathered}
$$

As can be seen in Figure 1(d), the period of the oscillation is four times the time taken by the oscillator to go from $u=0$ and $u=x_{0}^{2}$. Therefore,

$$
T=4\left(\frac{6}{3 q_{2}-\sqrt{-3 q_{0} q_{4}}}\right)^{1 / 2} K\left(m_{d}\right)
$$

where $m_{d}$ is given by

$$
m_{d}=\left(\frac{1}{2}-\frac{3 q_{2}}{2 \sqrt{-3 q_{0} q_{4}}}\right)^{-1} .
$$

$q_{0}, q_{2}$, and $q_{4}$ are defined in (19) and $K(m)$ is the complete elliptic integral of the first kind defined in (16). Figure 10 shows the period $T$ as a function of the initial amplitude $x_{0}$ (65) when $a_{1}=1,0<x_{0}<\left(-a_{1} / a_{5}\right)^{1 / 4}$ and (a) $a_{5}=-1$, (b) $a_{5}=-3$, (c) $a_{5}=-10$, and (d) $a_{5}=-50$. This figure corresponds to the right orbit in the phase space portrait shown in Figure 1(d). 
6.2. Calculation of the Exact Solution. In order to obtain the solution $x$ as a function of time $t$, from (64) it follows that

$$
\begin{aligned}
& F\left(\arcsin \sqrt{\frac{w_{1}\left(x_{0}^{2}-x^{2}\right)}{x_{0}^{2}\left(w_{1}-x^{2}\right)}}, \frac{x_{0}^{2}\left(w_{1}-w_{2}\right)}{w_{1}\left(x_{0}^{2}-w_{2}\right)}\right) \\
& =\sqrt{-\frac{1}{3} a_{5} w_{1}\left(x_{0}^{2}-w_{2}\right)} t
\end{aligned}
$$

$$
x(t)=\frac{x_{0} \operatorname{cn}\left(\left((1 / 2) q_{2}-(1 / 6) \sqrt{-3 q_{0} q_{4}}\right)^{1 / 2} t, m_{d}\right)}{\left[1+\left(2 /\left(1+\left(-3 q_{4} / q_{0}\right)^{1 / 2}\right)\right) \operatorname{sn}^{2}\left(\left((1 / 2) q_{2}-(1 / 6) \sqrt{-3 q_{0} q_{4}}\right)^{1 / 2} t, m_{d}\right)\right]^{1 / 2}} .
$$

It is easy to verify that (69) is also valid for trajectories $2 \rightarrow 3$ $\left(T / 4 \leq t \leq T / 2\right.$ and $\left.0 \geq x \geq-x_{0}\right), 3 \rightarrow 4(T / 2 \leq t \leq$ $3 T / 4$ and $\left.-x_{0} \leq x \leq 0\right)$, and $4 \rightarrow 1(3 T / 4 \leq t \leq T$ and $0 \leq x \leq x_{0}$ ), where the exact period $T$ is given in (65). It is important to point out that when $a_{1}>0$ and $a_{5}<0,(65)$ and (69) are valid provided that the initial amplitude $x_{0}$ satisfies the condition $0<x_{0}<\left(-a_{1} / a_{5}\right)^{1 / 4}$. Figure 11 shows the plot of the displacement $x$ as a function of time $t$ when $a_{1}=1, a_{5}=$ -3 , and $x_{0}=0.5$. This displacement was obtained using (69). This figure corresponds to the phase space portrait shown in Figure 1(d).

\section{Discussion}

In this section we briefly discuss the derived solutions presented in this paper compared to the exact one derived by Elías-Zúñiga [11], providing a discussion in which both solutions are compared for all the cases discussed in the manuscript. As it was pointed out in the introduction, ElíasZúñiga does not solve the nonlinear differential equation exactly as we have done here, but he assumes an expression for the solution. He proposed a solution for (1) with the initial conditions given in (2) which can be written as follows:

$$
x^{2}(t)=\frac{1}{a+b \mathrm{cn}^{2}(\omega t, m)} .
$$

Substituting (70) into (1) it is possible to obtain the values for $a, b, \omega$, and $m$, whose expressions in terms of $a_{1}, a_{5}$, and $x_{0}$ are

$$
\begin{aligned}
& a=-\frac{2 a_{5}}{a_{5} x_{0}^{2} \pm \sqrt{3} \sqrt{-a_{5}\left(4 a_{1} a_{5}+a_{5} x_{0}^{4}\right)}} \\
& b=\frac{1-a x_{0}^{2}}{x_{0}^{2}} \\
& m=\frac{1-a^{2} x_{0}^{4}}{1+2 a x_{0}^{2}}
\end{aligned}
$$

and then

$$
\begin{aligned}
& \frac{w_{1}\left(x_{0}^{2}-x^{2}\right)}{x_{0}^{2}\left(w_{1}-x^{2}\right)} \\
& \quad=\operatorname{sn}^{2}\left(\sqrt{-\frac{1}{3} a_{5} w_{1}\left(x_{0}^{2}-w_{2}\right)} t, \frac{x_{0}^{2}\left(w_{1}-w_{2}\right)}{w_{1}\left(x_{0}^{2}-w_{2}\right)}\right)
\end{aligned}
$$

and taking into account (19), (60), (66), and (68) we finally obtain

$$
\omega=\sqrt{-\frac{a_{1}+2 a a_{1} x_{0}^{2}}{1+a x_{0}^{2}+a^{2} x_{0}^{4}}} .
$$

Elías-Zúñiga also concluded that the corresponding exact period of oscillation $T$ of this nonlinear oscillator is given by [11, Eq. (15), page 2576]

$$
T=\frac{4 K(m)}{\omega}
$$

and pointed out that, depending on the system parameter values of $a_{1}, a_{5}$, and $x_{0}$, we can have real, complex, or imaginary values for $a, b, \omega$, and $m$. Now we are going to compare the exact period and solutions presented in this paper with Elías-Zúñiga's paper for all the cases discussed in the manuscript.

7.1. Exact Solution When $a_{1} \geq 0, a_{5}>0$, and $x_{0}>0$. We have obtained the exact period given in (17) and we have written the exact periodic solution of the quintic oscillator in the compact form given in (40). For this case, Elías-Zúñiga exact period is given in (72) and the exact solution has to be written as a piecewise function as follows:

$$
x(t)= \begin{cases}\left(a+b \mathrm{cn}^{2}(\omega t, m)\right)^{-1 / 2} & 0 \leq t \leq \frac{T}{4} \\ -\left(a+b \mathrm{cn}^{2}(\omega t, m)\right)^{-1 / 2} & \frac{T}{4} \leq t \leq \frac{3 T}{4} \\ \left(a+b \mathrm{cn}^{2}(\omega t, m)\right)^{-1 / 2} & \frac{3 T}{4} \leq t \leq T .\end{cases}
$$

When $a_{1}=1, a_{5}=3$, and $x_{0}=1$ we obtain

$$
\begin{aligned}
& a=-0.25 \pm 0.661438 i, \\
& b=1.25 \mp 0.661438 i, \\
& \omega=0.405233 \mp 1.63224 i, \\
& m=0.5625 \pm 0.826797 i
\end{aligned}
$$




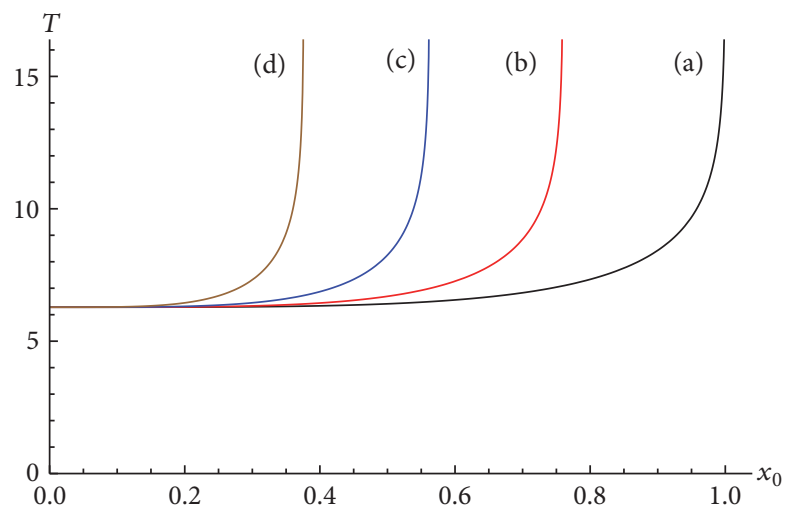

Figure 10: Exact period $T$ in (65) as a function of the initial amplitude $x_{0}$ when $a_{1}=1, x_{0}<\left(-a_{1} / a_{5}\right)^{1 / 4}$ and (a) $a_{5}=-1$, (b) $a_{5}=-3$, (c) $a_{5}=-10$, and (d) $a_{5}=-50$.

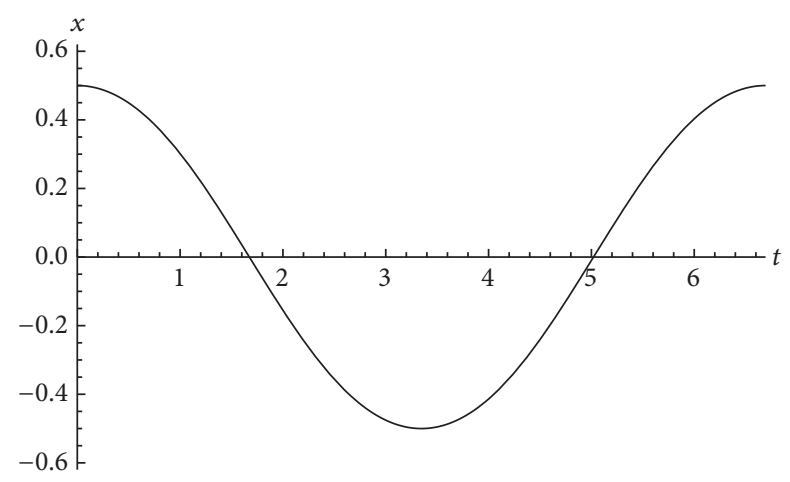

FIGURE 11: Exact solution $x$ in (69) as a function of time $t$ when $a_{1}=$ $1, a_{5}=-3$, and $x_{0}=0.5$.

and (72) gives for the period of this nonlinear oscillator the following value:

$$
T=1.89604 \pm 3.37321 i
$$

which is not a real number and cannot correspond to a period of an oscillatory system. The period we have obtained in this paper by using (17) is $T=3.79208 \mathrm{~s}$, which is the correct period. Substituting the values for $a, b, \omega$, and $m$ given in (74) into (73) and plotting $x$ as a function of time, we obtain Figure 3 , and it is possible to conclude that the solution given in (40) and the piecewise solution given in (73) are the same exact solution. However, the equation for the exact period given in (72) [11, Eq. (15), page 2576] does not give the exact period.

7.2. Exact Solution When $a_{1}<0, a_{5}>0$, and $x_{0}>$ $\left(-3 a_{1} / a_{5}\right)^{1 / 4}$. The exact period is given in (17) and the exact periodic solution is given in (40). For this case, Elías-Zúñiga exact period is given in (72) and the exact solution is written as a piecewise function given in (73).
When $a_{1}=-1, a_{5}=3$, and $x_{0}=1.1$ we obtain

$$
\begin{aligned}
a & =-1.3036 \pm 0.674788 i, \\
b & =2.13004 \mp 0.674788 i, \\
\omega & =0.171154 \mp 1.107 i, \\
m & =0.817588 \pm 0.575804 i
\end{aligned}
$$

and (72) gives for the period of this nonlinear oscillator the following value:

$$
T=2.8212 \pm 5.85337 i
$$

which is not a real number and cannot correspond to a period of a physical oscillatory system. The period we have obtained in this paper by using (17) is $T=5.64241 \mathrm{~s}$, which is the correct period. Substituting the values for $a, b, \omega$, and $m$ given in (76) into (73) and plotting $x$ as a function of time, we obtain Figure 5, and it is possible to conclude that the solution given in (40) and the piecewise solution given in (73) are the same exact solution. However, the equation for the exact period given in (72) [11, Eq. (15), page 2576] does not give the exact period.

7.3. Exact Solution When $a_{1}<0, a_{5}>0$, and $0<x_{0}<$ $\left(-3 a_{1} / a_{5}\right)^{1 / 4}$. We have obtained the exact period given in (49) and the exact periodic solution given in (52). For this case, Elías-Zúñiga exact period is given in (72) and the exact solution can be written as follows:

$$
x(t)=\frac{1}{\sqrt{a+b \mathrm{cn}^{2}(\omega t, m)}} .
$$

When $a_{1}=-1, a_{5}=3$, and $x_{0}=0.95$ we obtain

$$
\begin{aligned}
a & =-0.930194, \\
b & =2.03823, \\
\omega & =0.885853 i, \\
m & =-0.434817
\end{aligned}
$$

and (72) gives for the period of this nonlinear oscillator the following value:

$$
T=-6.46749 i
$$

which is an imaginary number and cannot correspond to a period of an oscillatory system. The period we have obtained in this paper by using (49) is $T=3.90327 \mathrm{~s}$, which is the correct period. Substituting the values for $a, b, \omega$, and $m$ given in (79) into (78) and plotting $x$ as a function of time, we obtain Figure 8(a), and it is possible to conclude that the solution given in (52) and the solution given in (78) are the same exact solution. However, the equation for the exact period given in (72) [11, Eq. (15), page 2576] does not give the exact period.

7.4. Exact Solution When $a_{1}>0, a_{5}<0$, and $0<x_{0}<$ $\left(-a_{1} / a_{5}\right)^{1 / 4}$. We have obtained the exact period given in (65) 
and the exact periodic solution given in (69). For this case, Elías-Zúñiga exact period is given in (72) and the exact solution is written as a piecewise function given in (73).

When $a_{1}=-1, a_{5}=3$, and $x_{0}=0.5$ we obtain

$$
\begin{aligned}
a & =1.1747, \\
b & =2.8253, \\
\omega & =1.07253 i, \\
m & =0.575648
\end{aligned}
$$

and (72) gives for the period of this nonlinear oscillator the following value:

$$
T=-7.80654 i
$$

which is an imaginary number and cannot correspond to a period of an oscillatory system. The period we have obtained in this paper by using (65) is $T=6.69369 \mathrm{~s}$, which is the correct period. Substituting the values for $a, b, \omega$, and $m$ given in (81) into (73) and plotting $x$ as a function of time, we obtain Figure 11, and it is possible to conclude that the solution we obtained (69) and the solution given in (73) are the same exact solution. However, the equation for the exact period given in (72) [11, Eq. (15), page 2576] does not give the exact period.

7.5. Why the Period Given in [11, Eq. (15), page 2576] Does Not Give the Exact Period? As we have previously pointed out, Elías-Zúñiga concludes that "since the Jacobian elliptic function $\mathrm{cn}(\omega t, m)$ has a period in $\omega t$ equal to $4 K(m)$, we may see that the corresponding exact period of oscillation $T$ is given by $T=4 K(m) / \omega "$ [11, page 2576]. As we have just proved, this equation for the period gives complex and imaginary values for the period of a physical oscillator but not real numbers. However, if Elías-Zúñiga's equations for the exact solution have been proven correct, where is the error in the expression for the period? The answer of this question is that not only the Jacobian elliptic function $\mathrm{cn}(\omega t, m)$ has a period in $\omega t$ equal to $4 K(m)$, but also periods $4 i K^{\prime}(m)$ and $2 K(m)+2 i K^{\prime}(m)$, where $K^{\prime}(m)=K(1-m)$ [22].

When $a_{1}=1, a_{5}=3$, and $x_{0}=1$, Elías-Zúñiga's period would have to be

$$
T=-\frac{4 i K^{\prime}(m)}{\omega}=-\frac{4 i K(1-m)}{\omega}=3.79208 \mathrm{~s}
$$

which gives the correct period. When $a_{1}=-1, a_{5}=3$, and $x_{0}=1.1$, Elías-Zúñiga's period would have to be

$$
T=-\frac{4 i K^{\prime}(m)}{\omega}=-\frac{4 i K(1-m)}{\omega}=5.64241 \mathrm{~s}
$$

which corresponds to the correct period for these numerical parameters. When $a_{1}=-1, a_{5}=3$, and $x_{0}=0.95$, the correct expression for Elías-Zúñiga's period would have to be

$$
\begin{aligned}
T & =\frac{-2 K(m)+2 i K^{\prime}(m)}{\omega}=\frac{-2 K(m)+2 i K(1-m)}{\omega} \\
& =3.90327 \mathrm{~s}
\end{aligned}
$$

which is the correct period. Finally, when $a_{1}=1, a_{5}=-3$, and $x_{0}=0.5$, the correct expression for Elías-Zúñiga's period would have to be

$$
T=\frac{4 i K^{\prime}(m)}{\omega}=\frac{4 i K(1-m)}{\omega}=6.69369 \mathrm{~s}
$$

which corresponds to the correct period for these parameters.

\section{Conclusions}

Closed-form expressions for the exact periods and solutions of the nonlinear quintic oscillator have been obtained for all possible oscillatory motions of this nonlinear system. Unlike Elías-Zúñiga's procedure [11, 12], we do not assume any expression for the solution but exactly solve the nonlinear differential equation, which allows us to obtain the period and, after inversion, the solution for this conservative nonlinear oscillator. As shown, there are four possible combinations of coefficients $a_{1}$ and $a_{5}$ which provide periodic motions. In three of them ((a) $a_{1} \geq 0, a_{5}>0$, and $x_{0}>0$, (b) $a_{1}<0$, $a_{5}>0$, and $x_{0}>\left(-3 a_{1} / a_{5}\right)^{1 / 4}>0$, and (d) $a_{1}>0$, $a_{5}<0$, and $\left.0<x_{0}<\left(-a_{1} / a_{5}\right)^{1 / 4}\right)$ the system oscillates around the equilibrium position $x=0$ with $x \in\left[-x_{0}, x_{0}\right]$. However, there is one more case ((c) $a_{1}>0, a_{5}<0$, and $0<$ $\left.x_{0}<\left(-3 a_{1} / a_{5}\right)^{1 / 4}\right)$ in which the system oscillates around the equilibrium position $x=\left(-a_{1} / a_{5}\right)^{1 / 4}$ with $x \in\left[x_{1}, x_{0}\right]$. In all cases the exact periods are given in terms of a complete elliptic integral of the first kind and the exact solutions are expressed in terms of Jacobi elliptic functions. We also showed that making a convenient change of variable to a new variable $y=x-\left(-a_{1} / a_{5}\right)^{1 / 4}$ it is possible to verify that case (c) is equivalent to a mixed-parity nonlinear oscillator oscillating around the equilibrium position $y=0$. Finally, our exact solutions are compared with the exact ones obtained by ElíasZúñiga [11].

\section{Competing Interests}

The authors declare that there is no conflict of interests regarding the publication of this paper.

\section{Acknowledgments}

This work was supported by the "Generalitat Valenciana" of Spain, under Project PROMETEOII/2015/015 and by the Universidad de Alicante, Spain, under Project GITE-09006UA.

\section{References}

[1] R. E. Mickens, Oscillations in Planar Dynamic Systems, World Scientific, Singapore, 1996.

[2] A. H. Nayfeh and D. T. Mook, Nonlinear Oscillations, WileyInterscience, New York, NY, USA, 1979.

[3] A. H. Nayfeh, Problems in Perturbation, John Wiley \& Sons, New York, NY, USA, 1985. 
[4] J. Lin, "A new approach to Duffing equation with strong and high order nonlinearity," Communications in Nonlinear Science and Numerical Simulation, vol. 4, no. 2, pp. 132-135, 1999.

[5] J. I. Ramos, "On Linstedt-Poincaré technique for the quintic Duffing equation," Applied Mathematics and Computation, vol. 193, no. 2, pp. 303-310, 2007.

[6] T. Pirbodaghi, S. H. Hoseini, M. T. Ahmadian, and G. H. Farrahi, "Duffing equations with cubic and quintic nonlinearities," Computers \& Mathematics with Applications, vol. 57, no. 3, pp. 500-506, 2009.

[7] B. S. Wu, W. P. Sun, and C. W. Lim, "An analytical approximate technique for a class of strongly non-linear oscillators," International Journal of Non-Linear Mechanics, vol. 41, no. 6-7, pp. 766-774, 2006.

[8] S. K. Lai, C. W. Lim, B. S. Wu, C. Wang, Q. C. Zeng, and X. F. He, "Newton-harmonic balancing approach for accurate solutions to nonlinear cubic-quintic Duffing oscillators," Applied Mathematical Modelling, vol. 33, no. 2, pp. 852-866, 2009.

[9] A. Beléndez, G. Bernabeu, J. Francés, D. I. Méndez, and S. Marini, "An accurate closed-form approximate solution for the quintic Duffing oscillator equation," Mathematical and Computer Modelling, vol. 52, no. 3-4, pp. 637-641, 2010.

[10] G. M. Scarpello and D. Ritelli, "Exact solution to a first-fifth power nonlinear unforced oscillator," Applied Mathematical Sciences, vol. 4, no. 69-72, pp. 3589-3594, 2010.

[11] A. Elías-Zúñiga, "Exact solution of the cubic-quintic Duffing oscillator," Applied Mathematical Modelling, vol. 37, no. 4, pp. 2574-2579, 2013.

[12] A. Elías-Zúñiga, "Exact solution of the quadratic mixed-parity Helmholtz-Duffing oscillator," Applied Mathematics and Computation, vol. 218, no. 14, pp. 7590-7594, 2012.

[13] A. Elías-Zúñiga, "Solution of the damped cubic-quintic Duffing oscillator by using Jacobi elliptic functions," Applied Mathematics and Computation, vol. 246, pp. 474-481, 2014.

[14] A. Elías-Zúñiga, "'Quintication” method to obtain approximate analytical solutions of non-linear oscillators," Applied Mathematics and Computation, vol. 243, pp. 849-855, 2014.

[15] A. Beléndez, T. Beléndez, F. J. Martínez, C. Pascual, M. L. Alvarez, and E. Arribas, "Exact solution for the unforced Duffing oscillator with cubic and quintic nonlinearities," Nonlinear Dynamics, vol. 86, no. 3, pp. 1687-1700, 2016.

[16] R. E. Mickens, Truly Nonlinear Oscillations, World Scientific, Singapore, 2010.

[17] I. Kovacic and Z. Rakaric, "Oscillators with a fractional-order restoring force: higher-order approximations for motion via a modified Ritz method," Communications in Nonlinear Science and Numerical Simulation, vol. 15, no. 9, pp. 2651-2658, 2010.

[18] S. K. Lai and K. W. Chow, "Exact solutions for oscillators with quadratic damping and mixed-parity nonlinearity," Physica Scripta, vol. 85, no. 4, Article ID 045006, 2012.

[19] S. L. Perko, Differential Equations and Dynamical Systems, Springer, New York, NY, USA, 1991.

[20] I. S. Gradshteyn and I. M. Ryzhik, Table of Integrals, Series and Products, Academic Press, San Diego, Calif, USA, 6th edition, 2000.

[21] L. M. Milne-Thomson, "Elliptic integrals", in Handbook of Mathematical functions with Formulas, Graphics and Mathematical Tables, M. Abramowitz and I. A. Stegun, Eds., chapter 17, pp. 587-607, Dover, New York, NY, USA, 1972.

[22] L. M. Milne-Thomson, "Jacobian elliptic functions and theta functions," in Handbook of Mathematical Functions with Formulas, Graphics and Mathematical Tables, M. Abramowitz and
I. A. Stegun, Eds., chapter 16, pp. 567-581, Dover, New York, NY, USA, 1972. 


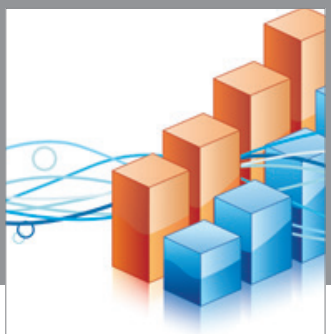

Advances in

Operations Research

vatem alat4

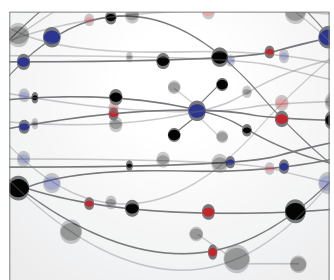

\section{The Scientific} World Journal
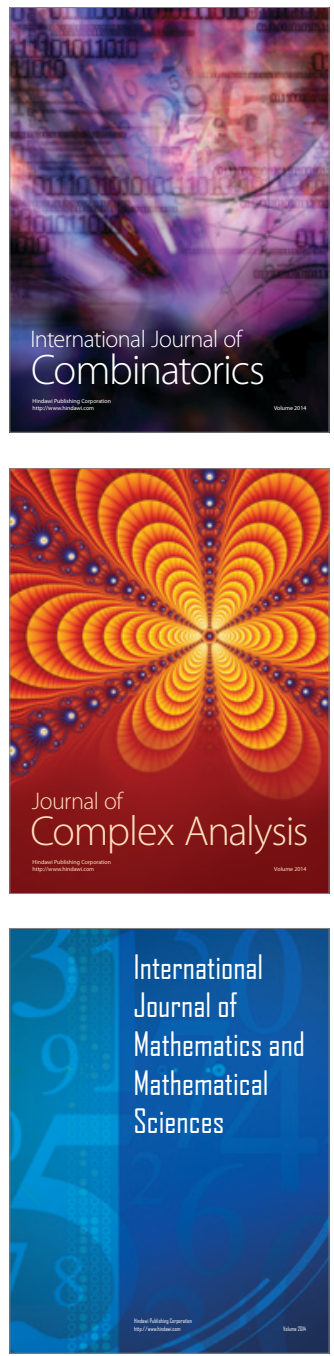
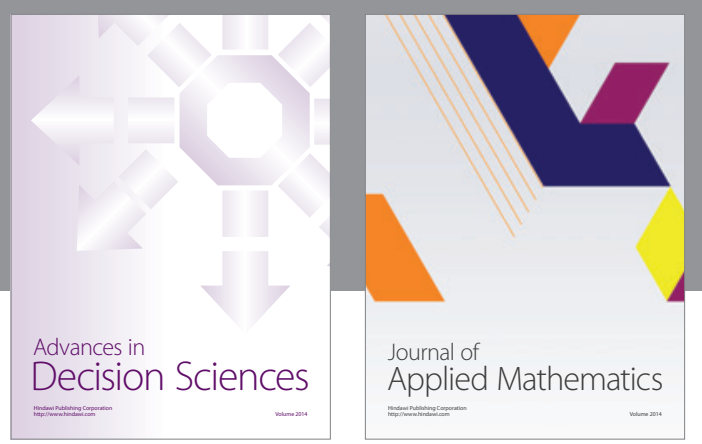

Algebra

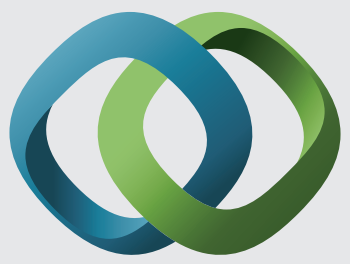

\section{Hindawi}

Submit your manuscripts at

https://www.hindawi.com
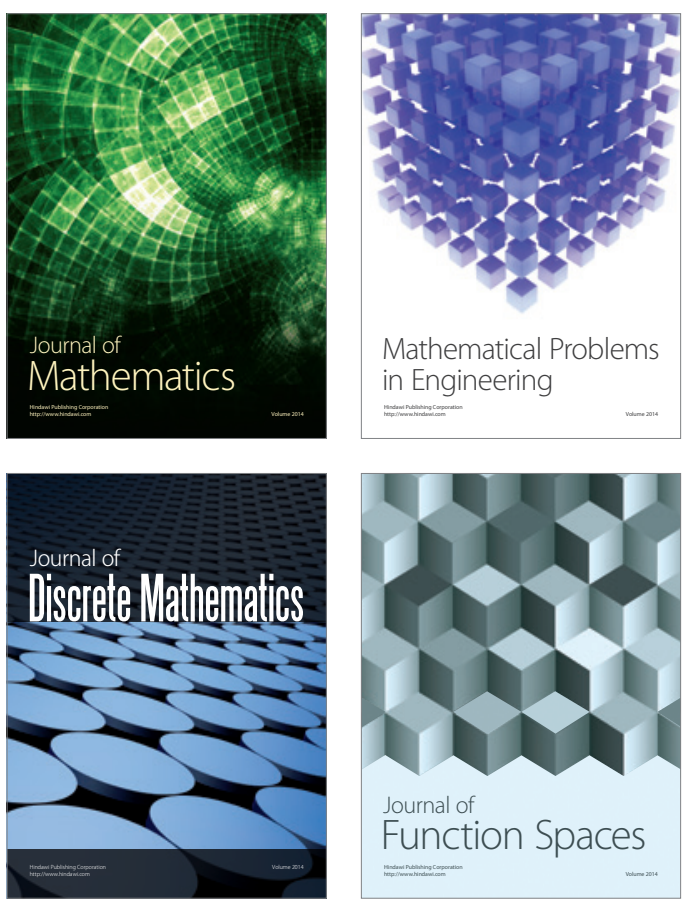

Mathematical Problems in Engineering
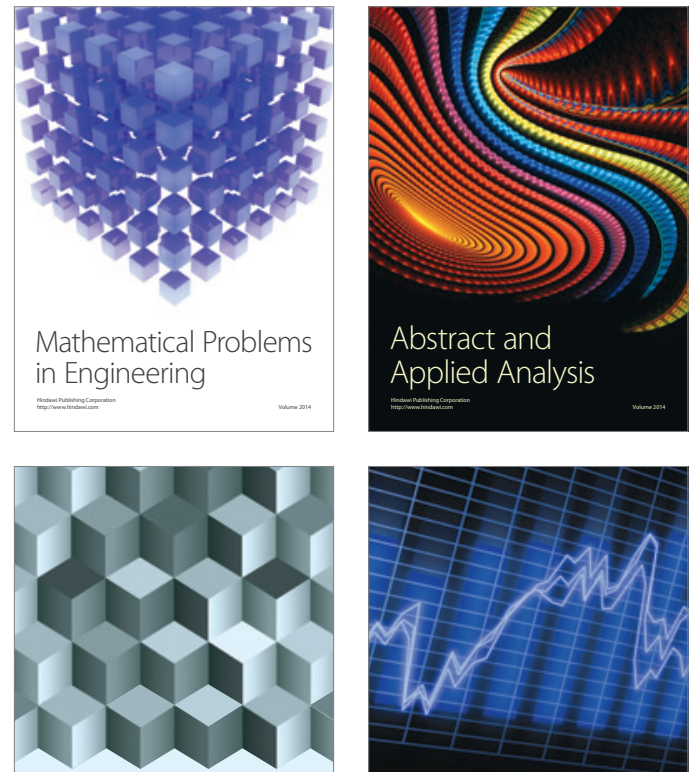

Journal of

Function Spaces

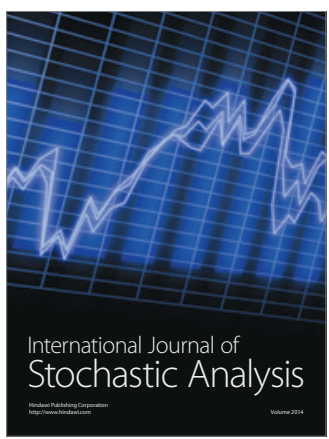

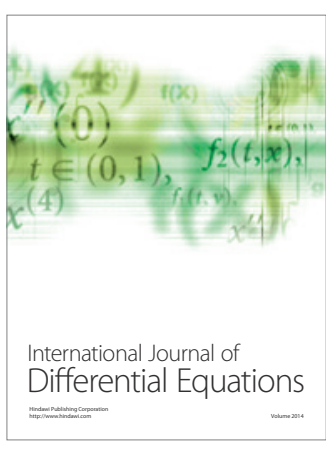
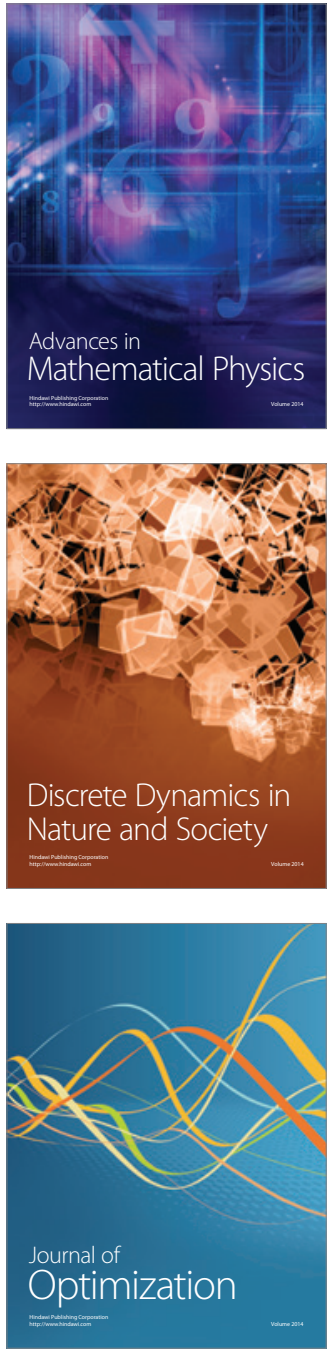